\title{
Nonlinear Ion-Acoustic Waves in Gravitating Dusty Plasma with Non-Isothermal Electrons and Fluctuating Dust Charges
}

\author{
S.N. PAul ${ }^{a}$, G. PAKIRA ${ }^{b}$, B. PAul ${ }^{a}$ And B. Ghosh ${ }^{a, *}$ \\ ${ }^{a}$ Department of Physics, Jadavpur University, Kolkata-700 032, India \\ ${ }^{b}$ Department of Physics, Sripat Sing College, Jiaganj, Murshidabad, W.B., India
}

(Received December 14, 2011; revised version April 4, 2012; in final form April 12, 2012)

\begin{abstract}
Using the reductive perturbation method a theoretical infrastructure has been developed to study the nonlinear propagation of ion-acoustic waves in self-gravitating multicomponent dusty plasma consisting of positive ions, non-isothermal electrons and negatively charged warm dust particles with fluctuating dust charges and drifting motion. It is shown that instead of coupled nonlinear equations as obtained by earlier authors the nonlinear propagation of ion-acoustic waves in such a plasma can be described by an uncoupled third order partial differential equation which is a modified form of the Korteweg-de Vries equation. From this equation, quasi-soliton solution is obtained for the ion-acoustic wave. The effects of non-isothermal electrons, gravity, dust charge fluctuations and drifting motion on the ion-acoustic solitary waves are discussed with application in astrophysical contexts. The importance of the model considered here has also been pointed out.
\end{abstract}

PACS: 52.27.Lw, 52.35.Fp, 04.40.-b

\section{Introduction}

During the past years dusty plasma has been a growing field of research because of its existence in various environments like cometary tails, planetary rings, asteroids, magnetosphere, lower ionosphere, interstellar and circumstellar clouds, laboratory devices, etc. The study of dusty plasma is relevant to the understanding of the problem of star formation. The probable cause of anomalous scattering of radio waves in the ionosphere may be due to the presence of dust grains in that region. Dusty plasma is a conglomeration of electrons, ions and $\mu \mathrm{m}-$ -sized dust grains. The mass of dust grains is typically about $10^{6}-10^{12}$ times the mass of an ion. The size of dust grains may vary in the range $0.05 \mu \mathrm{m}$ to $10 \mu \mathrm{m}$. Dust grains immersed in ambient plasmas are electrically charged by various processes. The charge on the dust grains influences their motion in electromagnetic fields and also affects the coagulation rate of dust into larger bodies. When the dust intergrain average separation is smaller than plasma Debye length, the collective rather than single-particle effects of dust grains become important.

It has been found both theoretically and experimentally that the presence of charged dust grains modifies the existing plasma wave spectra. Also in some cases

* corresponding author; e-mail: drsnpaul@gmail.com the presence of massive charged dust grains gives rise to new low-frequency eigenmodes of the dust electron-ion plasma.

The existence of dust-acoustic wave (DAW) was first predicted theoretically by Rao et al. [1], with the dust grains providing the inertia and the pressure of inertless electrons and ions providing the restoring force. The existence of dust-ion-acoustic wave (DIAW) was predicted by Shukla and Silin [2]. The existence of these new eigenmodes has also been confirmed experimentally [3, 4]. Rao [5] has also found the Alfven and magnetosonic modes in dusty plasma.

The presence of charged massive dust grains can significantly modify the linear and nonlinear wave propagation through plasma. When the size of the dust grains becomes considerable, the gravitational effects of dust grains become important though the effect is certainly negligible for electrons and ions. In fact, a number of authors have considered nonlinear wave propagation in self-gravitating dusty plasma where there is a competition between gravitational self-attraction and electrostatic repulsion between the charged grains, apart from other electromagnetic effects. It has been found that the gravitational effect can also significantly influence the nonlinear wave propagation through dusty plasma.

In magnetized dusty plasma with medium-sized grains the gravitational and magnetic effects may become comparable. It has been shown that self-gravitational effect in dusty plasma may lead to macroscopic instability of 
the Jeans type [6] which is believed to play a crucial role in the formation of many astrophysical objects such as galaxies, stars, etc. The magneto-gravitational instability of self-gravitating dusty plasma is relevant to the understanding of star formation. Thus the presence of charged dust grains in plasma influences wave propagation and various other collective properties of the plasma.

It is well known that dust particles get negatively charged due to attachment of the background electrons and ions on the surface via collisions [7]. The electrostatic charging of dust grains immersed in plasma is the main feature of dust-plasma interaction in dusty plasma. The charge on a dust particle does not remain fixed but depends on plasma properties, electron and ion currents flowing into or out of dust grains, photoemission, etc. In fact the charge on the dust grain can be considered as an extra dynamical variable which controls the motion of dust grains in fluctuating electrostatic fields. It has been shown that the charge fluctuation of the dust grains plays significant role on the dynamical behaviour of dusty plasmas [8-11]. Paul et al. [12] have studied the effects of streaming and attachment coefficients of ions and electrons on the formation of soliton in dusty plasma without considering the effects of gravity. They derived an inhomogeneous Korteweg-de Vries (KdV) equation and predicted the existence of a negative soliton in dusty plasma under some critical conditions and non-existence of solitons in dusty plasma with variable dust charge.

Most of the earlier works on solitons and shocks in dusty plasma are for the dust acoustic waves, though ion-acoustic solitons and shocks can also be excited in dusty plasma. Paul et al. [13] investigated the possibility of the existence of ion-acoustic solitary wave structures in gravitating dusty plasma with warm electrons, warm ions and cold dust particles having charge fluctuations. They showed that the nonlinear excitation of such waves follows a pair of coupled third order partial differential equations which is slightly different from the usual case of coupled $\mathrm{KdV}$ system; solitary wave structure is not possible in such self-gravitating dusty plasma with warm electrons and varying charges on the dust particles. However when the variation of charges of the dust particles is neglected, the solitary waves may be excited. The amplitude and width of such solitary waves are shown to be significantly changed by the gravitational effects on the dusty plasma.

In the study of ion-acoustic waves one usually considers Boltzmann distributed electrons. However this assumption cannot be maintained during the passage of waves in collisionless plasma. When the amplitude of the wave is large, electrons may be trapped in the potential trough [14]. In fact such trapping can occur even for small amplitude waves [15]. These trapped electrons interact strongly with the wave during the evolution of the wave and therefore cannot be treated on the same footing as the free electrons. Nonlinear wave structures in dusty plasma have been studied by several authors [16-20] including the effects of trapped electrons and ions. The electron-ion distributions play a crucial role in characterizing the physics of nonlinear waves [14, 21, 22]. They offer considerable increase in the richness and variety of wave motion in plasmas. They influence the condition for existence of nonlinear wave structures. Moreover, the presence of large amplitude waves can significantly modify the electron and ion distributions.

In recent years nonlinear wave structures have been studied by using different non-Maxwellian distributions such as non-thermal electrons [23], q-nonextensive electron velocity distributions [24-34]. El-Awady and Moslem [33] have studied the generation of nonlinear ion-acoustic waves in a plasma having nonextensive electrons and positrons. Tribeche et al. [34] have investigated arbitrary amplitude ion-acoustic solitary waves in a two-component plasma with $q$-nonextensive electrons. In fact it has been shown that for electrostatic wave propagation in plasma non-Maxwellian distribution presents a better fit to the experimental data while standard Maxwellian distribution only provides a crude description [35]. Recent studies show that the instability of self-gravitating plasma system is modified by the presence of non-Maxwellian effects [24, 30, 35]. In a self-gravitating plasma system where long-range interactions dominate and non-equilibrium stationary states exist, each particle is constantly feeling the influences by all other particles in the system, the energy distribution of the particles in the system is likely to be non-Maxwellian [36-38]. In fact self-gravitating plasma systems offer the best framework for searching into the effects of non-Maxwellian distributions on the properties of the system. The physics of self-gravitating dusty plasma is becoming increasingly relevant in determining the macroscopic behavior of extending systems in astrophysical scenario.

Non-isothermal distributions can be found during the condensation of dust grains in dusty plasmas. The presence of non-isothermal electrons in plasma gives rise to many interesting characteristics in nonlinear propagation of waves including the excitation of ion-acoustic solitary waves in plasma [39-42]. Observations of space plasmas indicate that the occurrence of non-Maxwellian electrons and ions is a common feature in such an environment. Thus it becomes important to study nonlinear propagation of ion-acoustic waves in self-gravitating dusty plasma including the effects of dust charge fluctuations as well as non-isothermal electrons.

The objective of the present paper is to study the existence and characteristics of ion-acoustic solitary waves in self-gravitating dusty plasma consisting of warm positive ions, non-isothermal electrons and dust particles with charge variations.

The paper is organized in the following way. In Sect. 2 we present the hydrodynamic equations for the ion and dust fluids, density distribution for the non-isothermal electrons, Poisson's equation for the electrostatic and gravitational potentials, and the equilibrium charge neutrality condition. Section 3 is devoted to the derivation of the modified $\mathrm{KdV}$ equation using reductive perturbation 
method. In Sect. 4 we find the solutions of the modified $\mathrm{KdV}$ equation. Finally in Sect. 5 we discuss the result with possible applications.

\section{Basic equations}

We consider an unmagnetized three-component plasma consisting of warm ions, warm $\mu \mathrm{m}$-sized massive negatively charged dust grains and non-isothermal electrons. In reality the size and mass of the dust grains may have different values. In our model plasma we assume that dust grains have uniform mass and behave like point charges. The charging of the dust particles is mainly caused by the attachment of the ions and electrons to the dust grains. The effects of photo-ionization radiation etc. for charging of the dust particles are neglected. Electrons because of their lighter mass and faster motion are initially attached to the dust particles at a faster rate than the ions. As a result dust particles get negatively charged. Once the dust particles get enough negative charge, the chance of an electron being attached to a negatively charged dust grain becomes much smaller than the chance of an ion being attached to the dust grain. So we may assume that the charge fluctuation of dust grains is due to the attachment of positive ions only. We consider the self-gravitating effect on the electrostatic dust acoustic wave in dusty plasma which is expected to influence both the linear and nonlinear modes of wave propagation. Under such assumptions, the set of normalized basic equations governing the plasma dynamics are the following $[43,44]$ :

For ions

$$
\begin{aligned}
& \frac{\partial n_{i}}{\partial t}+\frac{\partial}{\partial x}\left(n_{i} v_{i}\right)=-\beta_{i} n_{i} \\
& \frac{\partial v_{i}}{\partial t}+v_{i} \frac{\partial v_{i}}{\partial x}+\frac{\sigma_{i}}{n_{i}} \frac{\partial p_{i}}{\partial x}=-\frac{\partial \varphi}{\partial x}-\frac{\partial \psi}{\partial x}-\beta_{i}\left(v_{i}-v_{d}\right) \\
& \frac{\partial p_{i}}{\partial t}+v_{i} \frac{\partial p_{i}}{\partial x}+3 p_{i} \frac{\partial v_{i}}{\partial x}=0
\end{aligned}
$$

For dust particles

$$
\begin{aligned}
& \frac{\partial n_{d}}{\partial t}+\frac{\partial}{\partial x}\left(n_{d} v_{d}\right)=0, \\
& \frac{\partial v_{d}}{\partial t}+v_{d} \frac{\partial v_{d}}{\partial x}+\frac{\sigma_{i}}{\mu_{d} n_{d}} \frac{\partial p_{d}}{\partial x} \\
& =\frac{Z_{d}}{\mu_{d}} \frac{\partial \varphi}{\partial x}-\frac{\partial \psi}{\partial x}-\beta_{i}\left(v_{d}-v_{i}\right), \\
& \frac{\partial p_{d}}{\partial t}+v_{d} \frac{\partial p_{d}}{\partial x}+3 p_{d} \frac{\partial v_{d}}{\partial x}=0 .
\end{aligned}
$$

Poisson's equations

$$
\begin{aligned}
& \frac{\partial^{2} \varphi}{\partial x^{2}}=n_{e}-n_{i}+Z_{d} n_{d}, \\
& \frac{\partial^{2} \psi}{\partial x^{2}}=\frac{\omega_{g i}^{2}}{m_{i} \omega_{i}^{2}} \sum_{s=e, i, d} m_{s} n_{s} .
\end{aligned}
$$

Charge neutrality condition

$$
n_{i 0}=n_{e 0}+Z_{d} n_{d 0},
$$

where $m_{s}, n_{s}, v_{s}, q_{s}$ and $p_{s}$ denote respectively the mass, number density, velocity, charge and scalar pressure of the $s$-th species $(s=e, i, d)$ of plasma: $Z_{d}$ is the number of electronic charge attached on the grains; $\mu_{d}=m_{d} / m_{i}$, $\sigma_{i}=T_{i} / T_{e} ; n_{i 0}, n_{e 0}$ and $n_{d 0}$ are respectively the equilibrium number densities of ions, electrons and dust particles; $\beta_{i}$ is the attachment coefficient of the ions to the dust grain: $\varphi$ and $\psi$ are respectively the electrostatic and gravitational potentials; $\omega_{i}=\left(4 \pi e^{2} n_{i 0} / m_{i}\right)^{1 / 2}$ is the ion plasma frequency; $w_{g i}=\sqrt{4 \pi G m_{i} n_{i 0}}$ is the ion Jeans frequency, $G$ being the universal gravitational constant; other symbols have their usual significance. In the above equations we have normalized distance $x$ by the Debye length $\lambda_{\mathrm{D}}=\left(k_{\mathrm{B}} T_{e} / 4 \pi e^{2} n_{i 0}\right)^{1 / 2}$, time $t$ by the inverse of ion plasma frequency, all pressures by ion plasma pressure $n_{i 0} k_{\mathrm{B}} T_{i}, \varphi$ by $k_{\mathrm{B}} T_{e} / e, \psi$ by $k_{\mathrm{B}} T_{e} / m_{i}$ and all number densities by $n_{i 0}$. It may be noted that Eqs. (3) and (6) correspond to one - dimensional local adiabatic equation of state

$$
\frac{\mathrm{d}}{\mathrm{d} t}\left(p_{s} n_{s}^{-3}\right)=0
$$

in which the effects of viscosity, thermal conductivity and energy transfer due to collisions are neglected. Using the total time derivative operator as

$$
\frac{\mathrm{d}}{\mathrm{d} t}=\frac{\partial}{\partial t}+v_{s} \frac{\partial}{\partial x}
$$

and the equation of continuity one can easily establish Eqs. (3) and (6).

For the non -isothermally distributed electrons the number density is given by [45]:

$$
n_{e}=n_{e 0}\left(1+\varphi-\frac{4}{3} b \varphi^{3 / 2}+\frac{1}{2} \varphi^{2}+\ldots\right),
$$

where the constant $b$, called non -isothermal parameter, is given by

$$
b=\frac{1-\beta}{\pi^{1 / 2}},
$$

and the parameter $\beta$ is defined as

$$
\beta=T_{\text {ef }} / T_{\text {et }},
$$

in which $T_{\text {ef }}$ is the temperature for the free electrons and $T_{\text {et }}$ is the temperature for the trapped electrons. The parameter $\beta$ determines the nature of the distribution function giving a plateau if $\beta=0$ and a dip if $\beta<0$ and a hump if $\beta>0$. $\beta=1$ corresponds to Maxwellian distribution. In this paper the case $\beta \geq 0$ will be considered for the non -isothermal electrons.

\section{Derivation of the modified KdV equation}

For the derivation of the nonlinear equation governing the nonlinear dynamics of the wave, we make the usual stretching of the space coordinates and time [45],

$$
\xi=\varepsilon^{1 / 4}(x-V t) \text { and } \tau=\varepsilon^{3 / 4} t,
$$

where $V$ is the linear phase velocity and $\varepsilon$ is a smallness parameter measuring the dispersion and nonlinear 
effects. Further, we assume the following perturbation expansion for the field variables:

$$
\begin{aligned}
& X=X_{0}+\varepsilon X_{1}+\varepsilon^{3 / 2} X_{2}+\varepsilon^{2} X_{3}+\ldots, \\
& \beta_{i}=\varepsilon^{3 / 4} \overline{\beta_{i}},
\end{aligned}
$$

where $X$ stands for $n_{s}, v_{s}, p_{s}, \varphi, \psi$ with $\varphi_{0}=\psi_{0}=0$.

Using (13) and (14) in Eqs. (1)-(8) and equating the coefficients of the lowest order of $\varepsilon$ we obtain

$$
\begin{aligned}
& n_{i 1}=n_{i 0} \frac{v_{i 1}}{V-v_{i 0}}, \\
& v_{i 1}=\frac{1}{V-v_{i 0}}\left[a_{1} p_{i 1}+a_{2} \varphi_{1}+\psi_{1}\right], \\
& p_{i 1}=\frac{3}{V-v_{i 0}} v_{i 1}, \\
& n_{d 1}=n_{d 0} \frac{v_{d 1}}{V-v_{d 0}}, \\
& v_{d 1}=\frac{1}{V-v_{d 0}}\left[b_{1} p_{d 1}+b_{2} \varphi_{1}+\psi_{1}\right], \\
& p_{d 1}=\frac{3}{V-v_{d 0}} v_{d 1}, \\
& \varphi_{1}-n_{i 1}+Z_{d} n_{d 1}=0, \\
& 4 \pi G\left(m_{i} n_{i 1}+m_{d} n_{d 1}\right)=0,
\end{aligned}
$$

where

$$
\begin{aligned}
& a_{1}=\sigma_{i} / n_{i 0}, \quad a_{2}=1, \\
& b_{1}=\sigma_{i} / \mu_{d} n_{d 0}, \quad b_{2}=-Z_{d} / \mu_{d}
\end{aligned}
$$

and the mass of electron $m_{e}$ is neglected.

Using (15) and (16) in (17) we get

$$
A_{1} \psi_{1}=B_{1} \varphi_{1} \text {. }
$$

Similarly, from (18) we obtain

$$
A_{2} \psi_{1}=B_{2} \varphi_{1} \text {. }
$$

From (19) and (20) the linear dispersion relation for the ion-acoustic wave in self-gravitating dusty plasma is derived as

$$
A_{1} B_{2}-A_{2} B_{1}=0
$$

where

$$
\begin{aligned}
& A_{1}=\frac{n_{i 0}}{\lambda_{1}^{2}}-\frac{Z_{d} n_{d 0}}{\lambda_{2}^{2}}, \quad A_{2}=\frac{w_{g i}^{2}}{\lambda_{1}^{2}}+\frac{w_{g d}^{2}}{\lambda_{2}^{2}}, \\
& B_{1}=1-\frac{a_{2} n_{i 0}}{\lambda_{1}^{2}}+\frac{Z_{d} b_{2} n_{d 0}}{\lambda_{2}^{2}}, \\
& B_{2}=-\left(\frac{w_{g i}^{2}}{\lambda_{1}^{2}}+\frac{w_{g d}^{2} b_{2}}{\lambda_{2}^{2}}\right) \\
& \lambda_{1}=V_{1}^{2}-3 a_{1}, \quad \lambda_{2}=V_{2}^{2}-3 b_{1}, \\
& V_{1}=V-v_{i 0}, \quad V_{2}=V-v_{d 0}, \\
& w_{g d}=\sqrt{4 \pi G m_{d} n_{d 0}} \text { is the dust Jeans frequency. }
\end{aligned}
$$

$$
\lambda_{2}^{2} \omega_{g i}^{2}+\lambda_{1}^{2} \omega_{g d}^{2}=\left(1-b_{2}\right)\left(n_{i 0} \omega_{g d}^{2}+Z_{d} n_{d 0} \omega_{g i}^{2}\right) .
$$

It is a biquadratic equation in $V$ and its real positive solutions correspond to different modes of propagation. For a cold plasma $\sigma_{i}=0, a_{1}=b_{1}=0$, then with $v_{i 0}=$ $v_{d 0}=v_{0}$ one gets two modes of propagation given by

$$
\begin{gathered}
V=v_{0} \pm\left[\left(1-b_{2}\right)\left(n_{i 0} \omega_{g d}^{2}+Z_{d} n_{d 0} \omega_{g i}^{2}\right)\right. \\
\left./\left(\omega_{g i}^{2}+\omega_{g d}^{2}\right)\right]^{1 / 4} .
\end{gathered}
$$

Obviously one mode has relatively high phase speed and the other mode has relatively low phase speed. For a cold non-drifting plasma only one mode of propagation is possible.

Now, equating the coefficients of $\varepsilon^{7 / 4}$ we obtain from Eqs. (1)-(8):

$$
\begin{aligned}
& \frac{\partial n_{i 1}}{\partial \tau}+\bar{\beta}_{i} n_{i 1}=\left(V-v_{i 0}\right) \frac{\partial n_{i 2}}{\partial \xi}-n_{i 0} \frac{\partial v_{i 2}}{\partial \xi}, \\
& \frac{\partial v_{i 1}}{\partial \tau}+\bar{\beta}_{i}\left(v_{i 1}-v_{d 1}\right)=\left(V-v_{i 0}\right) \frac{\partial v_{i 2}}{\partial \xi} \\
& -a_{1} \frac{\partial p_{i 2}}{\partial \xi}-a_{2} \frac{\partial \varphi_{2}}{\partial \xi}-\frac{\partial \psi_{2}}{\partial \xi}, \\
& \frac{\partial p_{i 1}}{\partial \tau}=\left(V-v_{i 0}\right) \frac{\partial p_{i 2}}{\partial \xi}-3 \frac{\partial v_{i 2}}{\partial \xi}, \\
& \frac{\partial n_{d 1}}{\partial \tau}=\left(V-v_{d 0}\right) \frac{\partial n_{d 2}}{\partial \xi}-n_{d 0} \frac{\partial v_{d 2}}{\partial \xi}, \\
& \frac{\partial v_{d 1}}{\partial \tau}+\bar{\beta}_{i}\left(v_{d 1}-v_{i 1}\right)=\left(V-v_{d 0}\right) \frac{\partial v_{d 2}}{\partial \xi} \\
& -b_{1} n_{d 0} \frac{\partial p_{d 2}}{\partial \xi}-b_{2} \frac{\partial \varphi_{2}}{\partial \xi}-\frac{\partial \psi_{2}}{\partial \xi}, \\
& \frac{\partial p_{d 1}}{\partial \tau}=\left(V-v_{i 0}\right) \frac{\partial p_{d 2}}{\partial \xi}-3 \frac{\partial v_{d 2}}{\partial \xi}, \\
& \frac{\partial^{2} \varphi_{1}}{\partial \xi^{2}}=\varphi_{2}-\frac{4}{3} b \varphi_{1}^{3 / 2}-n_{i 2}+Z_{d} n_{d 2},
\end{aligned}
$$

and

$$
\frac{\partial^{2} \psi_{1}}{\partial \xi^{2}}=4 \pi G\left(m_{i} n_{i 2}+m_{d} n_{d 2}\right) .
$$

Using Eqs. (23)-(28) we derive the following modified $\mathrm{KdV}$ equation in gravitating dusty plasma containing non-isothermal electrons:

$$
P \frac{\partial \varphi_{1}}{\partial \tau}+Q \varphi_{1}^{1 / 2} \frac{\partial \varphi_{1}}{\partial \xi}+\frac{\partial^{3} \varphi_{1}}{\partial \xi^{3}}+R \varphi_{1}=0
$$

where

$$
\begin{aligned}
P & =\frac{n_{i 0}}{V_{1} \lambda_{1}}\left(a_{2}+\frac{A_{2}}{B_{2}}\right)\left(\frac{V_{1}^{2}}{\lambda_{1}}+\frac{3 a_{1} n_{i 0}}{\lambda_{1}}+1\right) \\
& +\frac{Z_{d} n_{d 0}}{V_{2} \lambda_{2}}\left(Z_{d}+\frac{A_{2}}{B_{2}}\right)\left(\frac{V_{2}^{2}}{\lambda_{2}}+\frac{3 b_{1} n_{d 0}}{\lambda_{2}}+1\right), \\
Q & =2 b, \\
R & =\frac{\overline{\beta_{i}} n_{i 0}}{\lambda_{1}}\left(a_{2} V_{1}+\frac{Z_{d} V_{2}}{\lambda_{2}}+\frac{a_{2}}{V_{1}}\right)+\frac{Z_{d}^{2} n_{d 0} V_{1} \overline{\beta_{i}}}{\lambda_{2}^{2}}
\end{aligned}
$$

Simplifying (21) we obtain 


$$
\begin{aligned}
& -\frac{Z_{d} n_{d 0} V_{1} \overline{\beta_{i}}}{\lambda_{2}^{2}} \frac{A_{2}}{B_{2}}+\frac{\overline{\beta_{i}} n_{i 0}}{\lambda_{1}}\left(\frac{V_{1}}{\lambda_{1}}+\frac{V_{2}}{\lambda_{2}}-\frac{1}{V_{1}}\right) \frac{A_{2}}{B_{2}} \\
& +\frac{\overline{\beta_{i}} n_{d 0} Z_{d}}{\lambda_{1} \lambda_{2}}\left(a_{2}+\frac{A_{2}}{B_{2}}\right) .
\end{aligned}
$$

If we neglect the effect of charge fluctuation on the dust grain, then $R=0$ and a solitary wave solution to Eq. (29) in a frame moving with velocity $\lambda$ can be obtained as

$$
\varphi_{1}=\left(\frac{15 P \lambda}{8 Q}\right)^{2} \operatorname{sech}^{4}\left(\frac{\sqrt{P \lambda}}{4}(\xi-\lambda \tau)\right) .
$$

Its amplitude and width depends on the non-thermal parameter and gravitational effect through $P$ and $Q$.

At this point we must mention that in the limit $\lambda_{1} \rightarrow 0$ or $\lambda_{2} \rightarrow 0$ the coefficients $A_{1}, A_{2}, B_{1}, B_{2}$ in Eq. (22) and the coefficients $P, R$ in Eq. (30) all diverge. So the evolution Eq. (31), derived by using perturbation technique, does not remain valid for values of $V$ for which $\lambda_{1} \rightarrow 0$ or $\lambda_{2} \rightarrow 0$. It corresponds to some sort of ion-acoustic resonance.

\section{Quasi-soliton solution}

$$
\begin{aligned}
& \text { Using } \tau / P=\eta \text { and }\left(\frac{8 Q}{15}\right)^{2} \varphi_{1}=\varphi \text { Eq. (29) becomes } \\
& \frac{\partial \varphi}{\partial \eta}+\frac{15}{8} \varphi^{1 / 2} \frac{\partial \varphi}{\partial \xi}+\frac{\partial^{3} \varphi}{\partial \xi^{3}}+R \varphi=0 .
\end{aligned}
$$

To solve Eq. (32) we note that the coefficient $R$ involving the attachment coefficients is practically small. With $R=0 \mathrm{Eq}$. (32) has a soliton solution

$$
\varphi=a_{0} \operatorname{sech}^{4}\left(\left(\frac{a_{0}}{16}\right)^{1 / 4}\left(\xi-\sqrt{a_{0}} \eta\right)\right),
$$

where $a_{0}$ is the amplitude, $\left(16 / a_{0}\right)^{1 / 4}$ is the width and $\sqrt{a_{0}}$ is the velocity. With $R \neq 0$ we also assume that Eq. (32) has a quasi-soliton solution

$$
\varphi=a(\eta) \operatorname{sech}^{4}\left(\left(\frac{a(\eta)}{16}\right)^{1 / 4}(\xi-\sqrt{a(\eta)} \eta)\right),
$$

whose amplitude can be expressed as [46]:

$$
a(\eta)=a\left(\eta_{0}\right) \exp \left(-R\left(\eta-\eta_{0}\right)\right),
$$

where $\eta_{0}$ corresponds to some initial value of the variable $\eta$.

Obviously if $R<0$, the amplitude of the soliton increases exponentially indicating instability. On the other hand, if $R>0$ the wave amplitude decays exponentially with the increase in the independent variable $\eta$.

\section{Results and discussions}

To describe the nonlinear propagation of ion-acoustic waves through self-gravitating dusty plasma we have derived an uncoupled third order nonlinear Eq. (29) which is a modified form of the usual $\mathrm{KdV}$ equation and includes the effects of gravity, dust charge fluctuations as well as that of non-isothermal electrons. It is important to note that instead of coupled nonlinear equations as obtained by earlier authors we have obtained an uncoupled equation which is advantageous. The model considered in the present paper can be easily applied to study the effects of different types of electron distributions on the nonlinear propagation of ion-acoustic waves in self-gravitating plasma. We have obtained the quasi-soliton solution (34) of the modified KdV Eq. (32).

The stability or instability of the soliton is found to depend on the sign of the coefficient $R$ of the fourth term $R \varphi$ in the modified $\mathrm{KdV}$ equation. The attachment coefficient, non-isothermal parameter and gravitational effects all enter into the factor $R$ in a complicated way. The stability or instability of the solitary structure will therefore be determined by the relative magnitudes of these effects. Thus the nonthermal parameter, the attachment coefficients and the gravitational effects involved in $R$ will significantly influence the soliton properties including soliton amplitude, speed, width as well as its stability. It is to be noted that the non-isothermality of the electrons has no effect on the linear properties of the wave but has significant effect on the nonlinear properties of the wave. For example, we note from Eqs. (31) and (30b) that the soliton amplitude decreases inversely as the square of the non-isothermal parameter $b$.

We have considered finite dust temperature in our analysis. Though in most astrophysical situations dusts can be considered as cold, there are some astrophysical and experimental situations where dust temperature may become comparable to ion temperature. The temperature of the dust particles is important owing to thermalization with the ions or orbital effects. In fact it has been suggested that the dust temperature in the planetary rings may have a large value compared to the electron temperature.

Inclusion of drift motion of plasma leads to two distinct modes of wave propagation - one having comparatively high phase speed and the other with lower phase speed. These modes may be called as "fast mode" and "slow mode", respectively. It is important to point out that instead of finding an uncoupled equation for $\varphi$ it is also possible to find an uncoupled equation for $\psi$ by following the same method as adopted in this paper. Then one can examine the possibility of having soliton-like variation of the gravitational potential.

To summarize, the most important achievement in this paper is that instead of coupled nonlinear equations as obtained by earlier authors an uncoupled nonlinear equation has been obtained which is advantageous. Also, the model considered here can be easily extended to study the effects of different types of electron distributions on the nonlinear propagation of ion-acoustic waves in self-gravitating dusty plasma.

\section{Acknowledgments}

We like to thank the reviewer for various suggestions and helpful comments in bringing the manuscript to the present form. 


\section{References}

[1] N.N. Rao, P.K. Shukla, M.Y. Yu, Planet Space Sci. 38, 543 (1990).

[2] P.K. Shukla, V.P. Silin, Phys. Scr. 45, 508 (1992).

[3] A. Barkan, R.L. Merlino, N. D'Angelo, Phys. Plasmas 2, 2563 (1995).

[4] N. Hayashi, Phys. Plasmas 8, 3051 (2001).

[5] N.N. Rao, Planet Space Sci. 41, 21 (1993).

[6] J.H. Jeans, Astronomy and Cosmology, Cambridge University Press, Cambridge 1998.

[7] N.F. Cramer, S.V. Vladimirov, Phys. Scr. 53, 586 (1996).

[8] J.B. Piper, J. Georee, Phys. Rev. Lett. 77, 3137 (1996).

[9] P.K. Shukla, G.T. Brik, G. Morfill, Phys. Scr. 56, 299 (1997).

[10] K.K. Mondal, Pramana J. Phys. 63, 1021 (2004).

[11] S. Ghosh, R. Bharuthram, M. Khan, M.R. Gupta, Phys. Plasmas 11, 3602 (2004).

[12] S.N. Paul, K.K. Mondal, A. Roychowdhury, Phys. Lett. A 257, 165 (1999).

[13] S.N. Paul, K. Roychowdhury, S. Burman, A. Roychowdhury, B. Paul, Czech. J. Phys. 56, 1453 (2006).

[14] Y.N. Nejoh, Phys. Plasmas 4, 2813 (1997).

[15] H. Schamel, Phys. Plasmas 7, 4831 (2000).

[16] C.K. Goertz, Rev. Geophys. 27, 271 (1989).

[17] D.A. Mendis, M. Rosenberg, Ann. Rev. Astron. Astrophys. 32, 419 (1994).

[18] M. Salahuddin, H.A. Shah, Phys. Scr. 69, 126 (2004).

[19] K. Avinash, P.K. Shukla, Phys. Lett. A 189, 470 (1994).

[20] P.K. Shukla, L. Stenflo, Proc. R. Soc. A 402, 403 (2006).

[21] S.K. El-Labany, W.F. El-Taibany, Phys. Plasmas 10 , 4685 (2003).

[22] S.K. El-Labany, W.F. El-Labany, A.A. Mamun, W.M. Moslem, Phys. Plasmas 11, 926 (2004).

[23] H. Alinejad, Astrophys. Space Sci. 327, 131 (2010).
[24] L. Liu, J. Du, Physica A 387, 4821 (2008).

[25] Z. Liu, L. Liu, J. Du, Phys. Plasmas 16, 072111 (2009).

[26] B. Sahu, Phys. Plasmas 18, 082302 (2011).

[27] P. Eslami, M. Mttaghizadeh, H.R. Pakzad, Phys. Plasmas 18, 102313 (2011).

[28] B. Sahu, Astrophys. Space Sci. 338, 251 (2012).

[29] P. Eslami, M. Mttaghizadeh, H.R. Pakzad, Astrophys. Space Sci. 338, 271 (2012).

[30] S. Shaikh, A. Khan, P.K. Bhatia, Phys. Lett. A 372 , 1451 (2008)

[31] M. Tribeche, L. Djebarni, Phys. Plasmas 17, 124502 (2010).

[32] H.R. Pakaz, Astrophys. Space Sci. 331, 169 (2011).

[33] E.I. El-Awady, W.M. Moslem, Phys. Plasmas 18 082306 (2011).

[34] M. Tribeche, L. Djebarni, R. Amour, Phys. Plasmas 17, 042114 (2010)

[35] J.A.S. Lima, R. Silva, A.R. Plastino, Phys. Rev. E 61, 3260 (2000).

[36] J. Du, Astrophys. Space Sci. 332, 47 (2007).

[37] J. Du, Phys. Lett. A 329, 262 (2004).

[38] J. Du, Europhys. Lett. 75, 861 (2006).

[39] R.A. Cairns, R. Binghan, R.O. Dendy, C.M.C Nairn, P.K. Shukla, A.A. Mamun, J. Phys. (Paris) 5, C 6 43 (1995).

[40] A.A. Mamun, Phys. Scr. 58, 505 (1998).

[41] A. Bandyapadhyay, K.P. Das, J. Plasma Phys. 62 , 255 (1999)

[42] S.K. Bhattacharya, S.N. Paul, Indian J. Phys. B 77, 327 (2003).

[43] R.K. Roy Chowdhury, S. Bhattacharya, Can. J. Phys. 65, 699 (1987)

[44] S.N. Paul, K.K. Mondal, A. Roy Chowdhury, Phys. Lett. A 257, 165 (1999).

[45] H. Schamel, J. Plasma Phys. 9, 377 (1973).

[46] W.S. Duan, J.B. Zhao, Phys. Plasmas 6, 3484 (1999). 